\title{
Особенности применения коаксиально-микрополосковых переходов для поверхностного монтажа типа SMP
}

А. Коренев ${ }^{1}$

УДК 621.3 | ВАК 05.27.01

\author{
Применение коаксиально-микрополосковых переходов (КМПП) для \\ поверхностного монтажа в СВЧ-трактах завоевывает все большую популярность \\ среди разработчиков современной радиоэлектронной аппаратуры, поскольку \\ позволяет не только снизить массогабаритные характеристики изделий, \\ но и повысить технологичность процесса сборки. В этой статье освещены \\ некоторые технические тонкости применения таких соединителей.
}

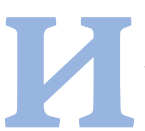

звестно, что точность передачи сигнала в СВчтракте в значительной степени зависит от применяемых компонентов и их монтажа [1]. Качество соединения возможно оценить при помощи таких характеристик как коэффициент стоячей волны по напряжению (КСВН) и вносимые потери. В рамках данной статьи было исследовано влияние посадочной площадки и качества пайки на характеристики коаксиально-микрополоскового перехода.

При проведении исследований был использован соединитель СР-50-973В (рис. 1) производства АО «Иркутский релейный завод". Тип соединителя - вилка угловая для поверхностного монтажа. Технические характеристики представлены в табл. 1. Существует три типоконструкции соединителя - с полным, ограниченным и скользящим сочленением. Они необходимы для построения переходов с платы на плату совместно с соединителями-переходами "розетка-розетка" (bullet), например СР-50-968ФВ (рис. 2) [2].

Рассмотрим влияние посадочной площадки на характеристики КМПП. При использовании соединителей СР-50-973В и их аналогов возникает необходимость в проектировании посадочной площадки, обеспечивающей получение минимального КСВН перехода с коаксиальной линии на печатную плату [3]. Проблема заключается в том, что производители этих соединителей часто не указывают, какие посадочные площадки должны быть использованы. Причиной сложившейся ситуации является сильная зависимость характеристик этих площадок от конструкции и материала печатной платы.

Для демонстрации этой зависимости была разработана посадочная площадка для материала Rogers RO4003C толщиной 0,508 мм с фольгой 35 мкм.

АО «Иркутский релейный завод", инженер-электроник,

korenev_av@irzirk.ru.
В качестве линии передачи на проектируемой печатной плате с соединителем СР-50-973В была использована копланарная линия с нижним слоем заземления. Такая линия обеспечивает наличие земли на поверхности печатной платы, что, с одной стороны, необходимо для монтажа соединителя, а с другой стороны, упрощает процесс проектирования перехода.

Конструкция копланарной линии должна быть такой, чтобы суммарная ширина центрального проводника и двух зазоров не превышала 1,4 мм, что соответствует зазору вокруг центрального проводника в соединителе (рис. 3). В нашем случае ширина центрального проводника равна 0,84 мм, а ширина зазоров-0,23 мм.

Посадочные площадки для печатной платы основываются на типовой конструкции (рис. 4). Данная конструкция показала себя наиболее универсальной при оптимизации ее размеров для применения с различными платами.

Основной задачей при проектировании посадочной площадки является устранение емкостной неоднородности, которая появляется в месте контакта центрального проводника соединителя с платой. Это достигается путем уменьшения ширин Wb и Wa согласующих участков

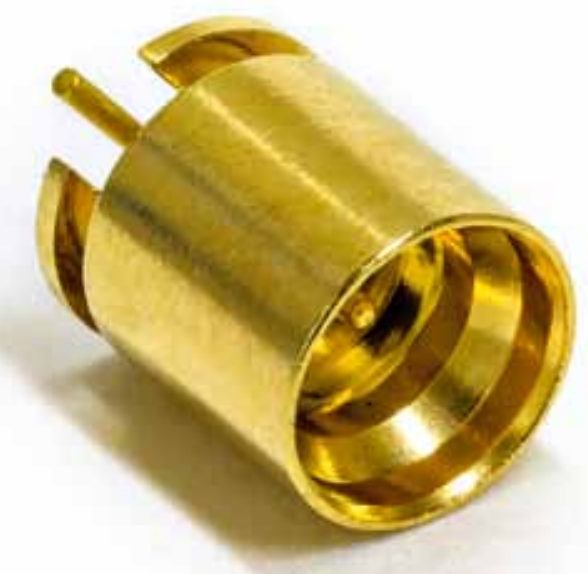

Рис. 1.

Внешний вид соединителя СР-50-973B 
(см. рис. 4) относительно ширины центрального проводника копланарной линии и выбора их длин (на рис. 4 - 0,4 и 2,15 мм). При этом суммарная длина отрезков не должна превышать длину вывода соединителя (2,85 мм, см. рис. 3). Суммарное значение 2,55 мм (в нашем случае) может быть использовано для большинства плат.

В табл. 2 приведены значения Wa и Wb согласующих отрезков для трех вариантов посадочной площадки. Первый вариант - плата без элементов согласования, второй вариант плата с упрощенной контактной площадкой и третий вариант - с оптимальной контактной площадкой для данной платы.

Второй и третий варианты посадочной площадки были разработаны с использованием пакета трехмерного электромагнитного моделирования. Для проектирования варианта с оптимальной контактной площадкой была использована модель соединителя на печатной плате, а для проектирования платы с упрощенной контактной площадкой был произведен расчет и оптимизация структуры, в которой поверх центрального проводника заземленной копланарной линии указанной конструкции находился цилиндр диаметром 0,4 мм и длиной 2,85 мм, имитирующий центральный проводник соединителя.

Для проведения измерений были разработаны три печатные платы, каждая с использованием своего варианта посадочной площадки (рис. 5). Расстояние между соединителями было выбрано равным 15 мм, чтобы, с одной стороны, обеспечить минимальную длину линии на плате для уменьшения потерь в ней, а с другой стороны, иметь возможность соединения при помощи штатных измерительных кабелей векторного анализатора цепей.
Земля на плате "прошита" переходными отверстиями для уменьшения индуктивности между верхним и нижним слоями. Диаметр площадки, свободной от паяльной маски (под пайку), равен 4,3 мм, но при необходимости может быть увеличен. Внешний вид платы с установленными соединителями представлен на рис. 6.

По результатам измерения и анализа S-параметров печатных плат (рис. 7) можно сделать несколько выводов:

- плата без согласования работоспособна только на частотах до бГГц;

- плата с оптимальным согласованием обладает хорошим КСВН $(<1,35)$ во всем диапазоне частот;

- плата с упрощенной посадочной площадкой обладает несколько худшими характеристиками, но, тем не менее, работоспособна во всем диапазоне частот и может быть использована в случаях, когда допустимо закрыть глаза на небольшое ухудшение характеристик.

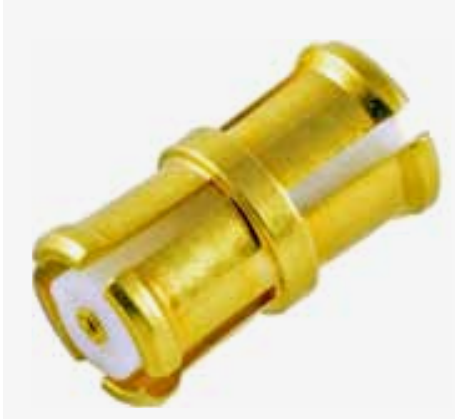

a)

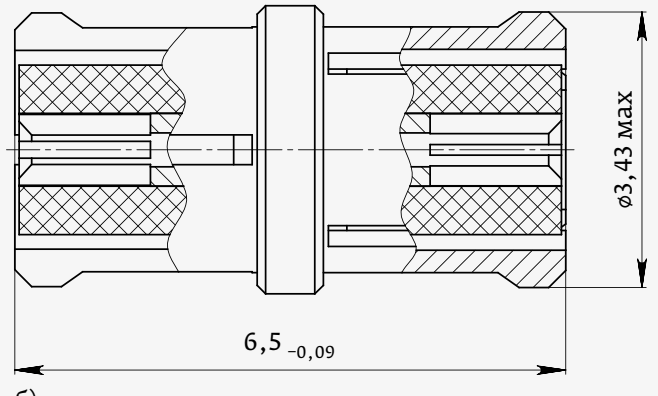

б)

Таблица 2. Значения размеров Wa и $\mathrm{Wb}$ трех посадочных площадок

\begin{tabular}{llll}
\hline № & $\begin{array}{l}\text { Посадочная } \\
\text { площадка }\end{array}$ & $\begin{array}{l}\mathrm{Wa}, \\
\mathrm{MM}\end{array}$ & $\begin{array}{l}\mathrm{Wb}, \\
\mathrm{MM}\end{array}$ \\
\hline 1. & $\begin{array}{l}\text { Без } \\
\text { согласования }\end{array}$ & 0,84 & 0,84 \\
\hline 2. & $\begin{array}{l}\text { Упрощенное } \\
\text { согласование }\end{array}$ & 0,55 & 0,55 \\
\hline 3. & $\begin{array}{l}\text { Оптимальное } \\
\text { согласование }\end{array}$ & 0,6 & 0,45 \\
\hline
\end{tabular}

Рис. 2. Внешний вид (а) и чертеж (б) соединителя СР-50-968ФВ 

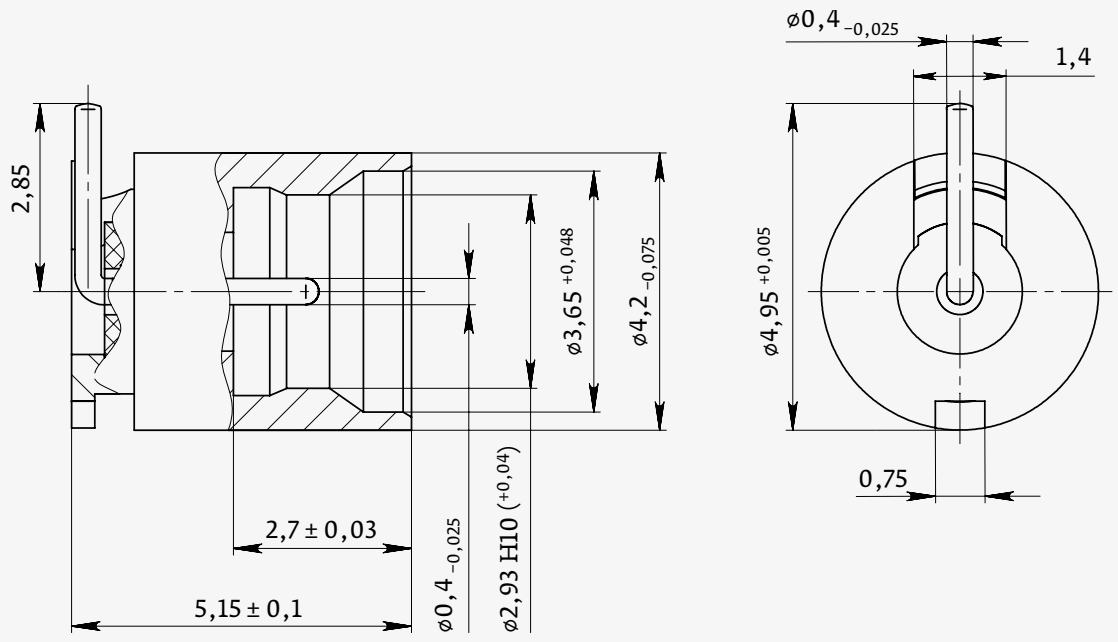

Рис. 3. Габаритный чертеж соединителя СР-50-973В (рис. 9). Образец № 1 имеет максимальный КСВН из трех измеренных, что объясняется наплывами припоя на корпусе вокруг центрального проводника, излишним количеством припоя на центральном проводнике и неточной ориентацией соединителя относительно копланарной линии. Все это создает значительную емкостную неоднородность, которая сводит на нет все усилия по оптимизации посадочной площадки. Образец № 2 имеет несколько лучший КСВН ввиду более точной ориентации на плате и меньшего количества припоя. Образец № 3 обладает отличными характеристиками и представляет собой эталон качественной пайки соединителя СР-50-973В.
Помимо контактной площадки, на КСВН перехода оказывает сильное влияние качество пайки соединителей. Для иллюстрации этой зависимости были измерены КСВН и вносимые потери соединителей, $\mathrm{cmoH}^{-}$ тированных по одному. На этот раз соединители были смонтированы на печатной плате, выполненной из материала Rogers RT/duroid 5880 толщиной 0,254 мм с фольгой 18 мкм. Wa и Wb для этого материала равны соответственно 0,55 и 0,4 мм. Данный материал является основным для проведения испытаний соединителей

Сопоставим графики зависимости КСВН переходов от частоты (рис. 8) с фотографиями паяного соединения

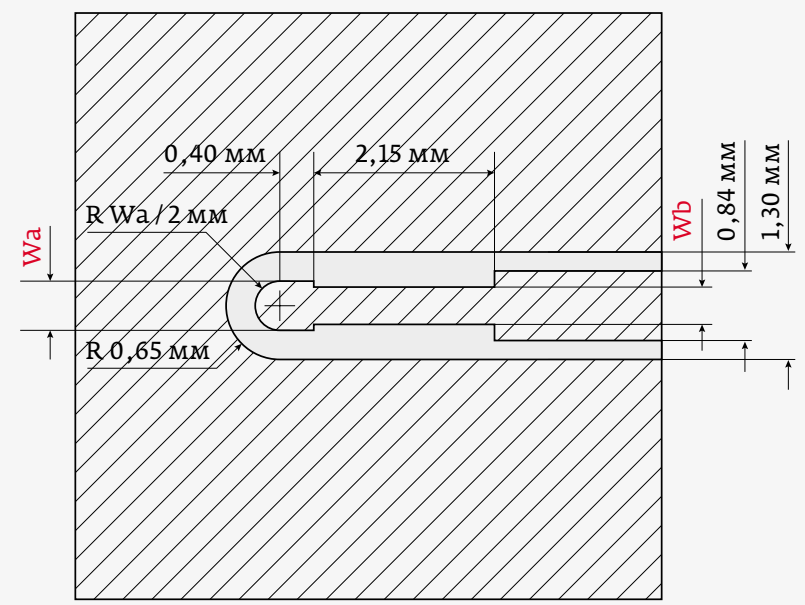

Рис. 4. Типовая конструкция посадочной площадки соединителя СР-50-973В

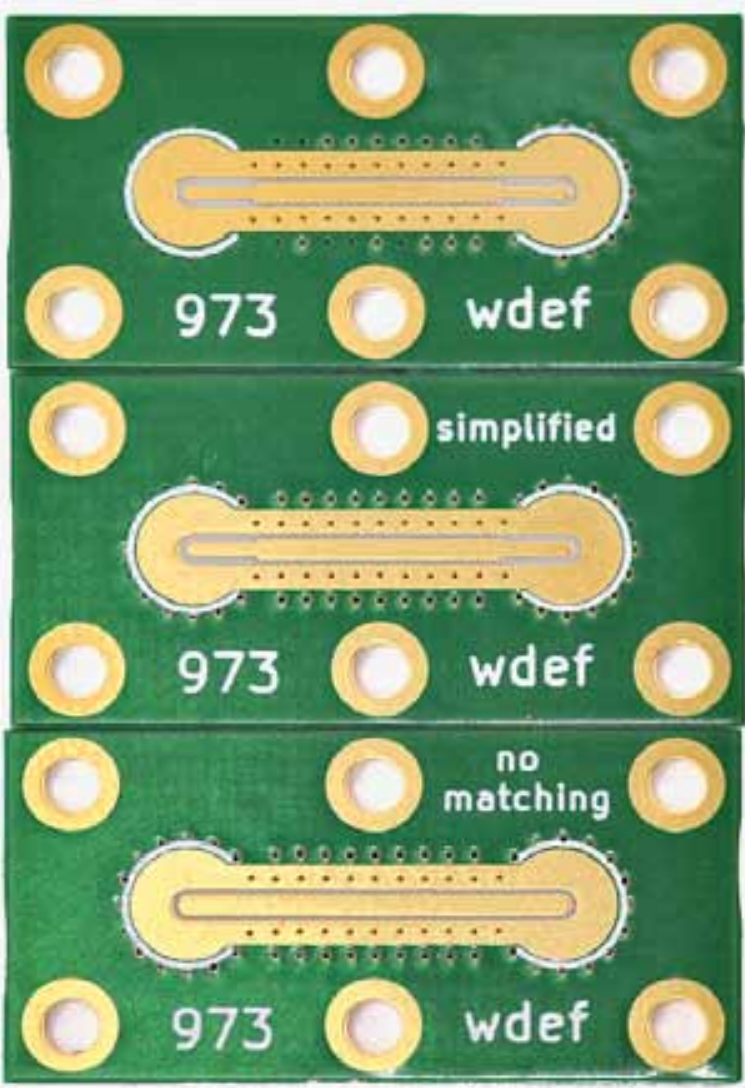

Рис. 5. Внешний вид разработанных вариантов плат. Вверху - с оптимальной посадочной площадкой, в середине - с упрощенной посадочной площадкой, внизу - с посадочной площадкой без согласования 


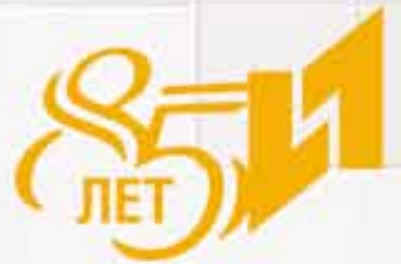

ИРКУТСКИЙ РЕЛЕЙНЫЙ

ЗАВОД

АКUИОНЕРКОЕ ОБЩЕСTHO

Россия, 664075, Иркутская обл., г. Иркутск, Тел.

ул. Байкапьская, 239

E-mail: marketing@irzirk.ru

www.irzirk.ru

приемная - (3952) 22-60-30

сбыт - тіф (3952) 24-76-19/ 24-57-45;

маркетинг - т/申 (3952) 35-23-18/ 24-56-46;

\section{AО «ИР 3 » предлагает к поставке изделия коммутационной техники}

\section{Реле электромагнитные}

Реле РЭКвз, РЭКвз В - неполяризованные, одностабипьные, герметичные постоянного тока, с двумя переключающими контактами
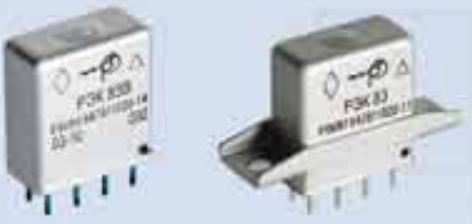

- Коммутируемый ток

до 3 А при индуктивной

нагрузке $\mathrm{C} \tau \leq 5 \mathrm{MC}$;

0.1 - 5.0 А постоянного тока:

$0.1-0.5$ A переменного тока.

- Коммутируемое напряжение

12 - 34 постоянного тока:

12 - 115 Взфф переменного тока.

- Гарантийный срок службы 25 лет.

Монтаж реле в аппаратуру

осуществляется пайкой на печатную

плату или за рамку еинтами.

Penе PЭС48, РЭС48 В - неполяризованные, одностабипьные, герметичные, постоянного тока, с двумя перекпючающими контактами.

Penе PЭС 48-1, РЭС48-1 В - с ппанарными выводами Аля поверхностного монтака на печатной плате (модификация реле РЭС48, РЭС48 В).

- Коммутируемый ток.

$10^{-6}$ - 3,0 A постоянного тока:

$0,1-1,5$ A переменного тока.

- Коммутируемсе напряжение.

$0,05-220$ B nостоянного тока:

12 - 150 Взфф переменного тока.

- Гарантийный срок спужбы 12 или 25 лет.

Тип монтажа реле; навесной, печапный, поеерхностный
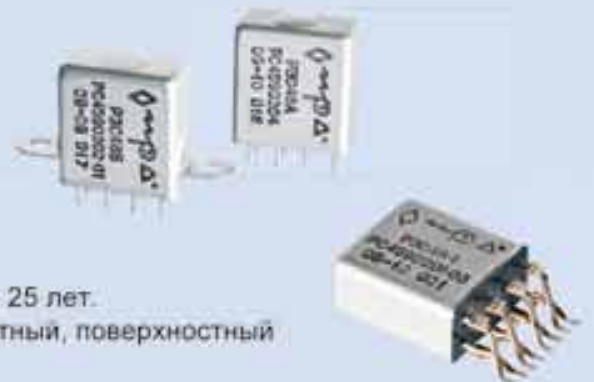

Радиочастотные соединители

Соединители радиочастотные врубные, герметичные и негерметичные КАПД. 434511.019 ТУ. Рабочий диапазон частот до 40 ГТч, присоединительные размеры типа SMP по стандарту MIL-STD-348B, Fig. 326.

\begin{tabular}{|c|c|c|}
\hline $\begin{array}{l}\text { Наименование } \\
\text { соединителя }\end{array}$ & Тип кабеля & $\begin{array}{c}\text { Внешний } \\
\text { вид }\end{array}$ \\
\hline \multicolumn{3}{|c|}{ Розетка кабельная прямая } \\
\hline CP- $50-969 \Phi B-00$ & $0,047^{*}$ & \\
\hline СР-50-969ФB-01 & $\begin{array}{l}\text { PK50-1-23, } \\
\text { PK50-1-24 }\end{array}$ & \\
\hline CP-50-969ФB-02 & $\begin{array}{c}0,086^{\prime \prime} \\
\text { PK50-1,5-22 }\end{array}$ & \\
\hline
\end{tabular}

Розетка кабельная угповая

\begin{tabular}{lc}
\hline CP-50-970ФB-00 & 0,047 \\
\hline CP-50-970ФB-01 & $\begin{array}{l}\text { PK50-1-23, } \\
\text { PK50-1-24 } \\
\text { CP-50-970ФB-02 }\end{array}$ \\
\hline $\begin{array}{c}0,086 \\
\text { PK50-1,5-22 }\end{array}$
\end{tabular}

Розетка кабельная прямая

\begin{tabular}{lc}
\hline CP-50-971ФB-00 & $0,047^{\circ}$ \\
\hline CP-50-971ФB-01 & $\begin{array}{l}\text { PK50-1-23, } \\
\text { PK50-1-24 }\end{array}$ \\
\hline CP-50-971ФB-02 & $\begin{array}{l}0,086^{\circ}, \\
\text { PK50-1,5-22 }\end{array}$ \\
\hline
\end{tabular}

Переход герметичный микрополосковый CPr-50-972B

\section{Bunka}

Anя nоверхностного монтажа CP-50-973B

Переход розетка-розетка CP- $50-968 \oplus B$

Переход герметичный вилка-виліка CPT.50-974B

Соединители радиочастотные с присоединительными размерами типа IX и SMA в соответствии с ГОСТ РB 51914-2002. Рабочий диапазон частот до $18 \mathrm{rTu}$

Вилки кабельные СР-50 ОКР кПереход-2w по разработке и освоению в производстве прямой и угловой кабельных вилок СР-50 Разработаны исполнения под спедующие типы кабеепя: PK-50-1, 5-22: PK-50-2-25: PK-50-3-28; PK-50-3-29; SR-085 RG-405; $0.086^{\prime \prime}, 0.113^{\prime \prime}, 0.141^{\prime \prime}$

\section{Соединители радиочастотные} герметичные СРГ-50 (КМПП) Подтверждена работоспособ̆ность издепий при пониженном атмосферном давлении $1,33 \cdot 10+7 \mathrm{Ta}$ $\left(1 \cdot 10^{-6}\right.$ мм рт. ст.) в установившемся режиме. Соответствуоцие изменения внесены в технические усповия ФИМД 430421.001 ТУ
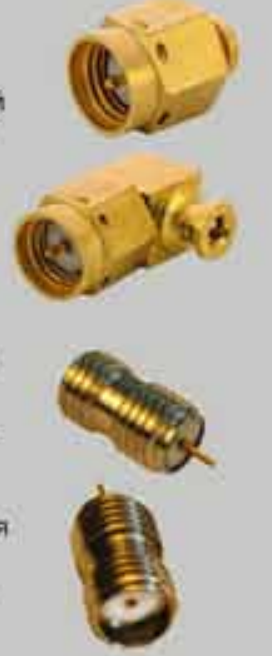

пыпеводонепроницаемьй перекnичатепь с безобрывным перекпючением контактов имеeт.

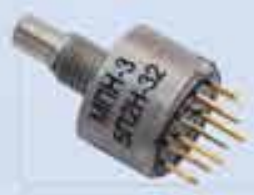
12 эл схем коммутации
- 3 runa sana yправпения
-4 варианта размера вылета
Пылеводонепроницаемый

Категория хачества - «ОТК» и шВПю 


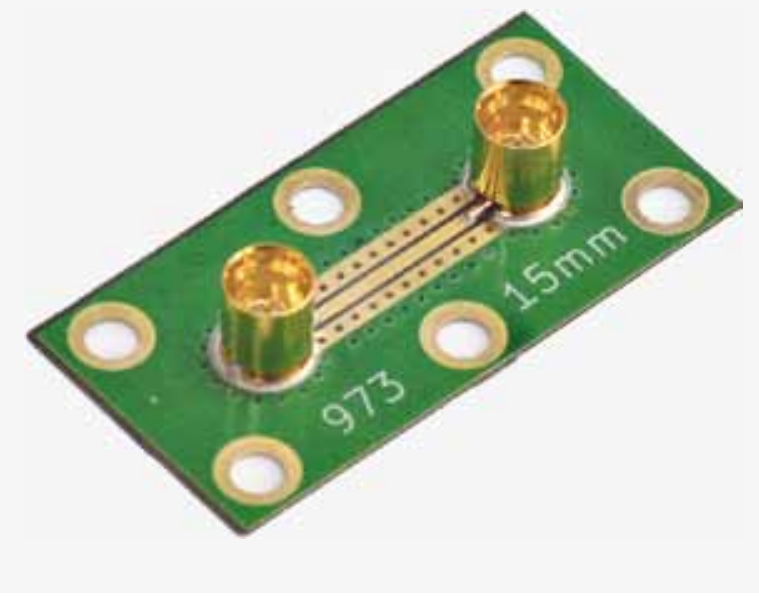

Рис. 6. Внешний вид платы с установленными соединителями

Для минимизации влияния пайки на КСBH перехода необходимо придерживаться следующих рекомендаций:

- недопускать натекания припоя на поверхность корпуса соединителя вокруг центрального проводника;

- не допускать излишнего количества припоя на центральном проводнике соединителя, особенно сверху;

- четко ориентировать соединитель на посадочной площадке.

Остановимся подробнее на методике измерений Анализ пар соединителей на печатной плате проводился с использованием векторного анализатора цепей (ВАЦ) Anritsu MS4644A, калибровка которого осуществлялась с помощью мер из состава калибровочного набора Rosenberger 19СК010-150. Этот набор - один из немногих доступных коммерческих калибровочных наборов с интерфейсом SMP. В качестве адаптеров 2,92 мм - SMP были использованы адаптеры Radiall R191.969.002.

Измерение параметров соединителей по отдельности производилось на измерительной оснастке для печатных плат Anritsu UTF 3680 в АО «НПП «Исток» им. Шокина» (рис. 10).

Опорная плоскость со стороны порта 1 анализатора была выбрана на границе перехода с оснастки на печатную плату, а со стороны порта 2 - на опорной плоскости адаптера 2,92 мм - SMP. Для получения такой конфигурации опорных плоскостей были проведены три полные двухпортовые калибровки - в сечениях 2,92 мм, SMP и измерительной оснастки.

Для калибровки в сечении 2,92 мм использовалось устройство для автоматической калибровки Anritsu 36585K

Для калибровки на оснастке был использован калибровочный набор Anritsu 36804В. Использованная

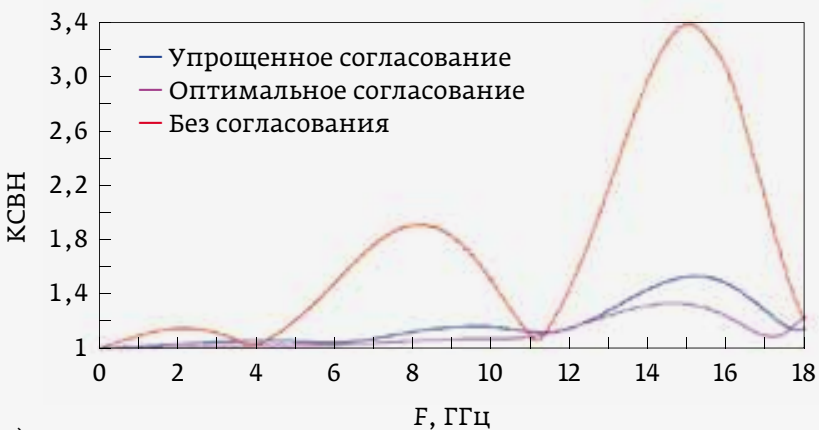

a)

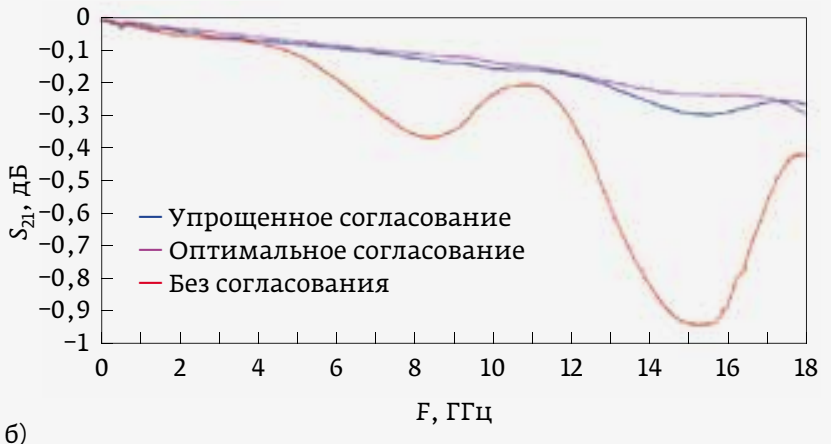

Рис. 7. Характеристики трех вариантов плат для пар соединителей: a - зависимость КСВН от частоты; б - зависимость вносимых потерь от частоты

калибровка - комбинация калибровок LRL и LRM с переходом на частоте 4,3 ГГц, что можно заметить по небольшому перелому на графике КСВН (см. рис. 8). Калибровочные меры LRL и LRM представляют собой набор отрезков микрополосковых линий передач разной длины (меры Line) и плату с двумя согласованными нагрузками на концах микрополосковых линий (меры Match). В качестве меры отражения (Reflect), задающей опорную плоскость, использовано

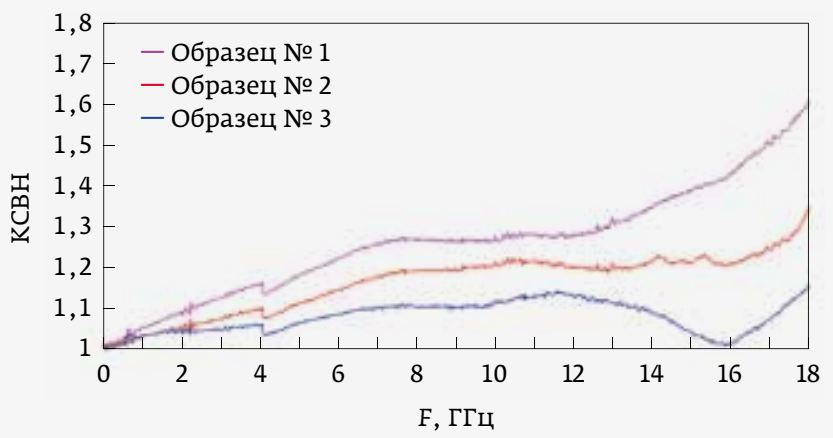

Рис. 8. Графики зависимости КСВН от частоты для трех соединителей в отдельности 

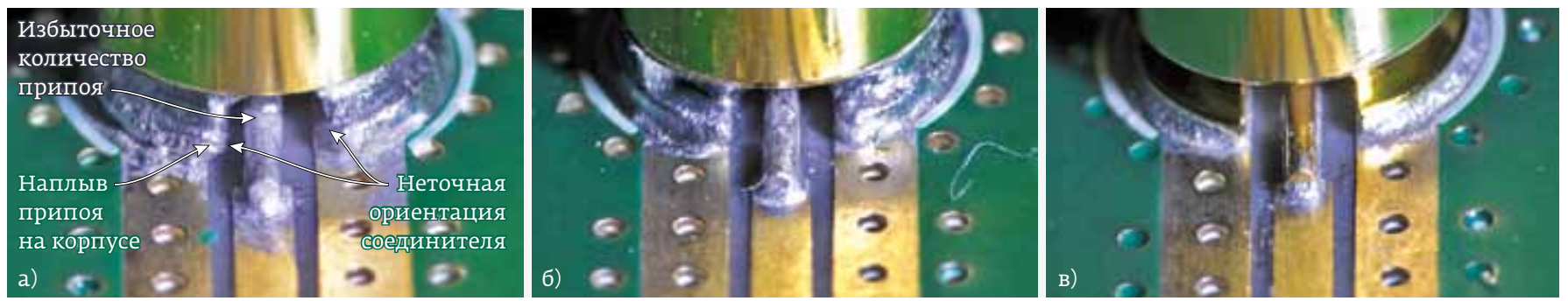

Рис. 9. Внешний вид соединителей, напаянных на печатную плату: а - образец № 1; 6 - образец № 2; в - образец № 3

соединение центрального проводника КМПП с зажимом оснастки, удерживающим печатную плату.

В настройках процедуры калибровки ВАЦ было выбрано два диапазона калибровки: LRM с линией длиной 10 мм и согласованной нагрузкой - от 10 МГц до 4,3 ГГц, и LRL с линиями длиной 10 и 12 мм - от 4,3 до 18 ГГц. Мера отражения в обоих случаях - мера К3 (Short-like Reflect). Детально процедура настройки калибровки и измерения калибровочных мер описана в [4].

Для получения S-параметров оснастки и переходов была использована функция "извлечение цепи типа С" (Network Extraction Tyре C) ВАЦ [5]. Эта функция позволяет, при наличии калибровок в двух опорных плоскостях, вычислить S-параметры четырехполюсников между ними. Для получения параметров переходов 2,92 мм - SMP были использованы сохраненные результаты калибровки в сечении 2,92 мм (наружная опорная плоскость - A) и в сечении SMP (внутренняя опорная плоскость - В). В результате работы алгоритма по определению параметров цепи получаются два файла с параметрами адаптеров, подключенных к портам 1 и 2. Аналогичным образом, с применением калибровок в сечениях 2,92 мм и измерительной оснастки, были получены характеристики двух половин UTF 3680. Детально процедура извлечения цепи (Network Extraction) описана в [6].

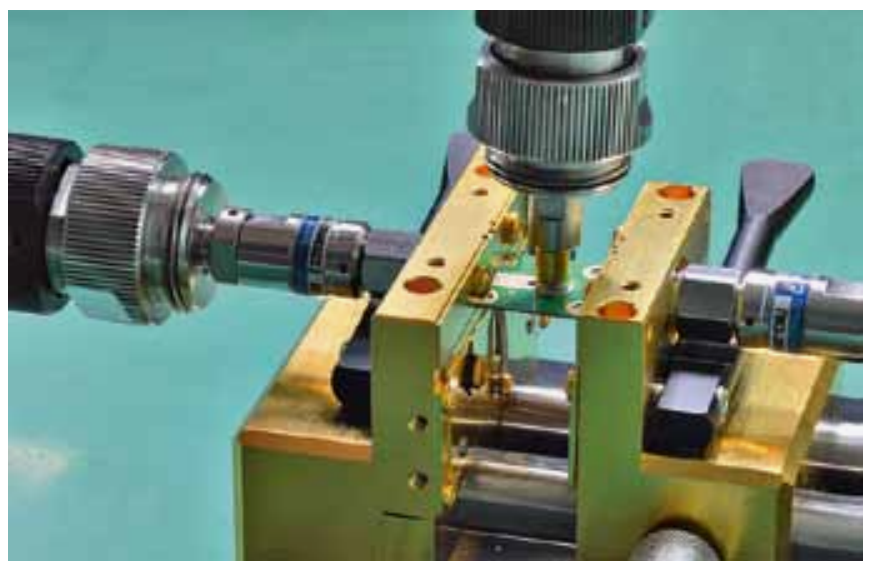

Рис. 10. Внешний вид измерительной установки в процессе измерения одного соединителя на печатной плате
Затем полученные характеристики были исключены из результатов измерения параметров соединителя на плате при помощи функции исключения цепи (De-embedding) ВАЦ. Порт 1 был подключен к измерительной оснастке (той же части, что и во время калибровки в сечении оснастки), поэтому для исключения цепи был выбран файл с параметрами части приспособления, подключенного к порту 1, а порт 2 - к адаптеру 2,92 мм - SMP (тому же, что и во время калибровки в сечении SMP), соответственно для исключения цепи был выбран файл с сохраненными характеристиками этого адаптера. Процедура исключения влияния цепи описана в [7].

$$
\text { औ. }
$$

Таким образом, в данной статье продемонстрирован процесс проектирования посадочных площадок для КМПП для поверхностного монтажа производства АО «Иркутский релейный завод". Также даны рекомендации по пайке этих соединителей. Следуя вышеизложенным методикам, можно добиться хороших параметров перехода в диапазоне частот до 18 ГГц.

\section{ЛИТЕРАТУРА}

1. Джуринский К.Б. Миниатюрные коаксиальные радиокомпоненты для микроэлектроники СВЧ / 2-е изд.М.: ТЕХНОСФЕРА, 2006.

2. Джуринский К., Чебунин М. Радиочастотные SMР-соединители. Новый взгляд // Компоненты и технологии. 2011 № 6. C. 34-40.

3. Corning Gilbert Inc., Microwave Push-on Interconnects / Corning Gilbert Inc. G-1500-102 05/11. P. 156-183.

4. Anritsu Company, 3680 Series Universal Test Fixture. Operation and Maintenance Manual. P/N 10410-00064H. 2013. P. 3-3.

5. Дансмор Дж. П. Настольная книга инженера. Измерения параметров СВЧ-устройств с использованием передовых методик векторного анализа цепей. - М.: ТЕХНОСФЕРА, 2019.

6. Anritsu Company, MS4640A Series Vector Network Analyzers. Calibration and Measurement Guide. P/N 10410-00269K. 2014. P. 8-15.

7. Anritsu Company, MS4640A Series Vector Network Analyzers. Calibration and Measurement Guide. P/N 10410-00269K. 2014. p. 10-3. 